\title{
Éxito educativo y condiciones socioeconómicas: los exámenes de habilitación para ejercer la medicina en Ecuador
}

\author{
Christian Escobar-Jiménez y Sergio Torres-Rentería
}

\section{RESUMEN}

Este trabajo analiza la relación entre los resultados del examen de habilitación profesional a graduados de medicina en Ecuador en 2017 y las condiciones socioeconómicas de los examinados. Se describen las variables involucradas en el instrumento de medición y los hallazgos por medio de estadísticos descriptivos, que muestran que los estudiantes provenientes de universidades privadas y cofinanciadas mantienen promedios significativamente más altos que los estudiantes de universidades públicas. Se considera también la trayectoria académica familiar como aspecto favorable para el éxito académico y profesional, medido en el nivel educativo del jefe del hogar. Se encuentra que la mayoría de graduados de medicina no son titulados de primera generación que ha accedido a la universidad, lo que podría ya determinar su éxito en la titulación con respecto a sus pares, en una de las tres carreras de más demanda en el país.

Palabras clave: sistema de educación superior, examen de habilitación para médicos en Ecuador, éxito profesional, éxito en educación superior, Ecuador.

Christian Escobar-Jiménez

cmescobar@puce.edu.ec Ecuatoriano. Doctor en Lógica y Filosofía de la Ciencia, Universidad de Santiago de Compostela, España; Doctorando en Sociología Analítica, Universidad Autónoma de Barcelona; Máster en Economía, EHEss-Francia; Máster en Relaciones Internacionales, UASB-Ecuador; Máster en Filosofía, UNED-España. Profesor auxiliar en la Escuela de Sociología y Ciencias Políticas de la Pontificia Universidad Católica del Ecuador (PUCE). Temas de investigación: filosofía de la ciencia, economía institucional, educación superior, filosofía del arte. ORCID: https://orcid.org/0000-0003-1940-2096. 


\title{
Êxito educativo e condições socioeconômicas: os exames de habilitação para exercer a medicina no Ecuador 2017
}

\section{RESUMO}

Este trabalho analisa a relação entre os resultados do exame de habilitação profissional a formandos de medicina no Ecuador em 2017 e as condições socioeconômicas dos examinados. Descreve-se as variáveis envolvidas no instrumento de medição e as descobertas por meio de estatísticas descritivas, que mostram que os estudantes provenientes de universidades privadas e cofinanciadas mantém medias significativamente mais altas que os estudantes de universidades públicas. Se considera também a trajetória acadêmica familiar como aspecto favorável para o êxito acadêmico e profissional, medido no nível educativo do chefe do lar. Se encontra que a maioria de formandos de medicina não são graduados de primeira geração que acederam à universidade, o que poderia já determinar seu êxito na formação com respeito a seus pares, em uma das três carreiras de mais demanda no país.

Palavras chave: sistema de educação superior, exame de habilitação para médicos no Ecuador, êxito profissional, êxito em educação superior, Ecuador.

\section{Educational success and socioeconomic conditions: the qualifying exams to practice medicine in Ecuador 2017}

\begin{abstract}
This paper analyzes the relationship between the results of the professional habilitation exam to medical graduates in Ecuador in 2017 and the socioeconomic conditions of the examinees. The variables involved in the measurement instrument and the findings are described by means of descriptive statistics, which show that students coming from private and co-financed universities maintain significantly higher averages than students from public universities. The family academic trajectory is also considered as a favorable aspect for academic and professional success, measured by the educational level of the head of household. It is found that the majority of medical graduates are not first-generation graduates, which could already determine their success in graduating with respect to their peers, in one of the three most in-demand careers in the country.
\end{abstract}

Key words: higher education system, qualifying examination for physicians in Ecuador, professional success, success in higher education, Ecuador. 


\section{Introducción}

Este trabajo se enfoca en el análisis de las calificaciones obtenidas durante 2017 en el Examen de Habilitación para el Ejercicio Profesional (en adelante EHEP) de los graduados de medicina en Ecuador. Este estudio vincula la relación entre éxito educativo y características socioeconómicas de los graduados de medicina, a partir de las calificaciones y los datos arrojados por las encuestas levantadas a los candidatos como requisito para examinarse.

Los estudios que relacionan éxito educativo y características socioeconómicas han sido prolíficos, aunque, por lo general, se han enfocado en el análisis de la escolaridad media y básica, sobre todo en con un enfoque que enlaza condiciones socioeconómicas desfavorables y resilencia ${ }^{1}$ o repitencia (Harris, 2007; Cordero et al., 2015); o en el nivel de ingresos familiares y éxito escolar (Blanden y Gregg, 2004; Carrillo et al., 2018); o estudios que relacionan capacidad intelectual, éxito escolar y lactancia materna (Victora et al., 2015); o factores culturales y éxito escolar (Harris III et al., 1976), o desde una perspectiva multifactorial (Clark et al., 2006).

Frecuentemente, la mayor parte de estos trabajos estudian el éxito educativo en su propia trayectoria, es decir, tomando como variable dependiente calificaciones, aprobación de cursos, diplomas o carreras y como factores explicativos a variables socioeconómicas. En el caso de este estudio, quienes se someten al examen de habilitación ya han obtenido su título profesional en medicina, es decir, el examen es un punto medio entre la graduación (un primer factor o momento, que puede ser entendido ya como éxito escolar) y la posibilidad de habilitarse o calificarse y obtener la licencia de ejercicio de la medicina. La aplicación de este tipo de exámenes también existe en otros países de la región, como Perú y Chile; y en países como Colombia y México existen mecanismos de evaluación externos de las carreras médicas y sus estudiantes, con miras al ejercicio profesional. Para el caso ecuatoriano, el examen aplicado por The National Board of Medical Examiners de Estados Unidos ha sido el principal referente institucional para la evaluación previa al ejercicio. Existen análisis de los instrumentos aplicados para Chile (Enríquez y Mena, 2005), Ecuador (Ruano et al., 2018), Estados Unidos (Subhiyah, 2018). En general, la lógica de este tipo de exámenes intenta conectar conocimientos teóricos, capacidad de análisis de casos concretos y aplicación práctica de esos conocimientos. Tomando en cuenta la naturaleza técnica de la medicina, los exámenes se enfocan en los aspectos prácticos, a pesar de que se aplican de forma escrita.

En sentido institucional, la aplicación de este examen es obligatoria a partir de la Ley Orgánica de Educación Superior 2010. Este instrumento es desarrollado y aplicado por el Consejo de Aseguramiento de la Calidad de la Educación Superior (CACES), y busca valorar las competencias adquiridas durante el proceso de formación universitaria, acreditando de modo objetivo que el profesional cuenta con una formación suficiente para el desenvolvimiento en su campo laboral, en aquellas carreras que pudieran comprometer el interés público, es decir, aquellas carreras que ponen en riesgo la vida, la salud y la seguridad de la ciudadanía (medicina, odontología, enfermería). En la elaboración del examen confluye el Ministerio de Salud Pública (MPS) y las instituciones de educación superior, pues se toman en cuenta tanto las competencias profesionales como las de graduación.

\section{Discusión teórica}

Los estudios clásicos que establecen una relación entre crecimiento económico e inversión en educación se han enfocado generalmente en lo que se conoce

\footnotetext{
${ }^{1}$ Se entiende por resiliencia a la capacidad de los estudiantes de inserción, reinserción y desarrollo de sus capacidades a pesar de las condiciones desfavorables. Cuando un estudiante cursa más de una vez un mismo año lectivo, hablamos de repitencia.
} 
como formación del capital humano. Por un lado, estos estudios concluyen que la inversión en capital humano está directamente relacionada con el progreso económico; por otro, reconocen que el entorno crea los incentivos para la adquisición de habilidades y conocimientos para las elecciones personales sobre educación (Coleman, 1968; Becker, 1994; Noguera, 2009). Sin embargo, sobre la teoría del capital humano se han tejido varias revisiones y objeciones, relacionadas a las inequidades que causa una desigual distribución del acceso a la educación a pesar de buenos impactos intergeneracionales; la estructura socioeconómica y el acceso, además de la elección de estudios que se valoran más en un momento determinado, crear mecanismos de sobrecompensación (Deaton, 2015). Según Cadil et al. (2014) la nueva evidencia señala que la inversión en capital humano no está necesariamente conectada ni garantiza el crecimiento económico, pues, además de la cualificación, es necesaria la demanda efectiva y la capacidad instalada para colocar a los profesionales.

A más de las condiciones económicas que predicen que familias con mejores ingresos tienen hijos con mayores probabilidades de éxito académico, existen factores culturales, de trayectoria, apoyo familiar y predisposición individual para el éxito. El nivel educativo de los padres suele ser favorable para el éxito escolar (Coleman et al., 1968; Yang y Gustaffson, 2004; Cordero et al., 2015). El estudio de las condiciones socioeconómicas en el impacto de las calificaciones obtenidas se suele usar como forma de medir la igualdad de un sistema educativo (Cordero et al., 2015).

De la misma manera, en sociedades altamente diferenciadas en cuanto a la existencia de etnias o nacionalidades, también se analiza como una variable para explicar aspectos de acceso público, sub-representación, acceso a educación privada o pública. Por ejemplo, en Estados Unidos, la población afrodescendiente está estigmatizada desfavorablemente en términos culturales, o tiene otro tipo de problemas concretos, como mayores niveles de enfermedades crónicas, posibilidad de muerte en el primer año de vida, de ser despedido, menor expectativa de vida, probabilidades de ser declarado culpable, etcétera. Este mismo tipo de aspectos también se relacionan con la educación, como mayores probabilidades de ser clasificados por retardo mental, de asistir a algún tipo de forma de educación especial o menos éxito en calificaciones (Noguera, 2003; 2009; Scott, Taylor y Palmer, 2013). En este mismo país, por ejemplo, se ha constatado que la estratificación social crea escuelas mayoritariamente de población afrodescendiente que funcionan como guetos que perpetúan estigmas y formas de fracaso escolar (Coleman, 1966; Noguera, 2009). Otros estudios demuestran tendencias similares para el caso de latinos en Estados Unidos (Crisp, Taggart y Nora, 2015). En España, este clase de estudios se han centrado en el éxito de la población migrante, cuyos estudiantes suelen presentar también peores promedios generales, ateniéndose también a cómo los factores culturales inciden en los problemas de inserción y éxito educativo (Pàmies Rovira, 2011; Lorenzo et al., 2012).

En general, los trabajos sobre educación superior suelen presentar varios tipos de diferencias, pues se debe tener en cuenta que la trayectoria misma implica tamices o filtros que de a poco van restringiendo el acceso. En este sentido, se suele tener en cuenta perspectivas multifactoriales. A las condiciones socioeconómicas se suman variables psicológicas o el propio interés del área de estudios (O’Neil et al., 2011; Brint y Clotfelter, 2016). Mientras la educación básica puede ser considerada como un derecho subjetivo, connatural a las personas, asociado a los derechos humanos, la educación superior se concibe como un derecho objetivo (Escobar-Jiménez y Delgado, 2019), lo que limita accesos por inversión pública. Estas variables no socioeconómicas también marcan diferencias sustanciales en el éxito educativo, sobre todo si se tiene en cuenta en un área como medicina, en la que los factores de éxito, deserción y fracaso se han analizado más que desde la 
perspectiva socioeconómica, desde los requisitos de acceso previos, los valores otorgados por la familia, la educación familiar, el entorno cultural y la motivación personal (O’Neil et al., 2011).

Para el caso de América Latina, varios estudios muestran cómo persiste una limitación estructural sobre el acceso, relacionado a formas de reforzamiento de la exclusión social, como se demuestra para el caso colombiano (Sverdlik, Ferrari y Jaimovich, 2005). En el caso ecuatoriano, a pesar de una mejora sostenida en el acceso entre los quintiles más bajos, la desigualdad de acceso estructural persiste debido a los filtros de ingreso a la educación superior a pesar de la gratuidad. En este caso, aunque se ha dado una disminución de la desigualdad en el acceso, la inequidad estructural persiste (Ponce y Carrasco, 2016). En general, en América Latina, la educación superior se sostiene todavía como un sistema de élites (Chiroleu, 2009), que permite que también haya una diferencia sustancial en la demanda de profesionales en universidades privadas de mayor prestigio, como pueden ser los casos chileno, ecuatoriano, colombiano o peruano, a pesar de un creciente acceso a la educación superior pública. Por ejemplo, Rama (2009) señala este aspecto como un problema que marca el carácter en el acceso y la masificación de la educación superior en América Latina.

Por otro lado, en un estudio sobre la equidad en la educación superior en la región (García de Fanelli y Jacinto, 2010) se evidencia que: 1) el acceso a la educación superior está marcado por filtros estructurales, asociados a la pobreza o la ubicación geográfica, como sucede en las zonas rurales de Perú o Bolivia, lo que ya limita el acceso hasta en dos tercios; 2) si en las ciudades mejora el acceso, no supera el tope de $50 \%$, pero no hay diferencias de género marcadas; 3) el éxito educativo está concentrado en los quintiles más altos; 4) la mayoría de estudiantes de la región son primeras generaciones que acceden a la universidad. Este último aspecto, por ejemplo, se observa también en el caso ecuatoriano, del cual se trata en este estudio, con respecto a los graduados de medicina.

\section{Descripción de los datos}

Los datos utilizados provienen de la Encuesta de Evaluados aplicada a las personas que rindieron el Examen de Habilitación para el Ejercicio Profesional (EHEP) de la carrera de medicina correspondiente al periodo 2017, por parte del CACES. ${ }^{2} \mathrm{La}$ Encuesta de Evaluados recoge información sobre las características socioeconómicas de los hogares de los examinados. Las variables que se incluyen en este estudio son individuales: sexo, edad, etnia; características de los hogares: financiamiento de los estudios, nivel de escolaridad de los padres, acceso a servicios públicos, a bienes y enseres del hogar.

Los datos fueron recabados entre marzo y julio de 2017. El número total de evaluados fue de 5 139, solo 22 personas más con respecto a los 5107 que se presentaron en 2016. En el presente estudio se trabaja con 4736 evaluados nacionales, lo que representa el 92\% del total. Cabe recalcar que el 8\% restante son extranjeros (de mayoría venezolana). Se descarta esta cantidad para obtener datos más consistentes con respecto a la trayectoria y el núcleo familiar en Ecuador. De la misma forma, la sección de la encuesta que corresponde a percepción no fue tomada en cuenta dados los fines del presente estudio.

Con base en los datos de acceso a servicios públicos y a bienes para uso del hogar (sobre todo tecnológicos), se construyeron dos variables simples para describir la cobertura y acceso de los hogares de los examinados, sobre acceso a necesidades básicas y el nivel de acceso a bienes enseres. Estas variables se encuentran definidas como el número de bienes

\footnotetext{
${ }^{2}$ El Examen de Habilitación para el ejercicio Profesional (EHEP) es una evaluación que tiene por finalidad acreditar, de modo objetivo, que el profesional cuenta con una formación suficiente para el desenvolvimiento en su campo laboral, y que se constituye como un requisito para ejercer la profesión en el país.
} 
disponibles (básicos y bienes-enseres) en los hogares de los examinados en 2017.

La descripción de los evaluados incluye aspectos individuales: sexo, edad, autoidentificación étnica, discapacidad, motivación para la elección de la carrera, continuidad o interrupción de los estudios, tipo de financiamiento para el pago de matrícula, manutención en el transcurso de los estudios y empleo o actividad económica remunerada en el transcurso de los estudios.

Entre las características del hogar se encuentran: tamaño, materiales de la construcción de la vivienda, acceso a agua, electricidad y alcantarillado, enseres que registra la vivienda y número de miembros del hogar que realizan actividad económica. En estas variables se encuentra también la descripción del nivel educativo de los jefes de hogar. ${ }^{3}$ Finalmente, la información de la encuesta permite explorar la forma en la que contribuye el acceso a bienes tanto públicos como muebles y enseres, sobre el rendimiento educativo de los evaluados. Las variables creadas para ello son:

- Cobertura de necesidades básicas: este índice va de 0 a 6 , los valores se van acumulando a medida que el hogar puntúa un bien más. Los criterios para esta medida están estandarizados en encuestas como las del Instituto Ecuatoriano de Estadísticas y Censos, y son: materiales de techo, paredes y piso, electricidad, agua potable, alcantarillado. El índice se acumula por cada servicio o bien del hogar.

- Enseres del hogar: este índice va de 0 a 5, los valores se van acumulando a medida que el hogar puntúa un bien más. En este caso, no se trata de bienes o servicios básicos, sino del acceso a bienes enseres que mejoran la calidad de vida, el tiempo para el desarrollo de tareas domésticas y también las actividades escolares. Estos bienes enseres son: televisor, ordenador (de escritorio o portátil), teléfono inteligente, automóvil, acceso a internet. De la misma forma, el índice va acumulando un punto más a medida que el hogar tiene uno de estos bienes enseres.

\section{Metodología}

Por un lado, este trabajo tiene una aproximación descriptiva sobre las características socioeconómicas de los estudiantes y sus hogares. Los aspectos que se abordan son: género, escolaridad del jefe de hogar, tipo de financiamiento de los estudios, índice de necesidades básicas satisfechas e índice de bienes enseres del hogar. Se agrupa a los estudiantes por el tipo de financiamiento de la universidad en la cual se graduaron. Existen tres grupos posibles: pública, privada y cofinanciada (universidades privadas que reciben en parte financiamiento del Estado).

Dada la literatura que relaciona condiciones socioeconómicas y éxito educativo, los promedios de calificaciones entre universidades privadas y públicas debería variar. Para esto se usarán comparaciones por diferencias de medias bajo la prueba $\mathrm{t}$ de Student, puesto que las distribuciones entre los diferentes grupos son normales en cuanto a los resultados del examen.

Por otra parte, hubo una aproximación inferencial intentando aplicar un modelo de regresión con el método de máxima verosimilitud con variables dicotómicas (modelo probit), en el que se consideran dos grupos, quienes aprobaron el examen (1) y quienes lo reprobaron (0). La variable dicotómica es dependiente, mientras las variables independientes son las socioeconómicas, generalmente, de tipo categórico (índice de servicios y bienes enseres, apoyo económico de los padres durante los estudios, etcétera). Los resultados del modelo no permitieron establecer una relación entre los factores socioeconómicos y los resultados de aprobación o no aprobación. Este problema se discute en las conclusiones de este estudio.

\footnotetext{
${ }^{3}$ En el caso de jefe de hogar no necesariamente es el padre.
} 


\section{Descripción de variables}

La tabla del Anexo muestra que, para un total de 4 736 evaluados, el $74.6 \%$ aprobó el examen con un promedio de 64.05 , con notas que varían entre 32 como mínima y 87 máxima, con una desviación estándar de 6.54. Por otro lado, se corrobora que la medicina es una carrera básicamente femenina, con el $61 \%$, mostrando una tendencia similar en 2016 . La moda del año medio de nacimiento es 1988, lo que sitúa en los 29 años a la mayoría de los examinados, con una desviación estándar de 3.77. En cuanto a la identificación étnica, el 89\% de los examinados se califican a sí mismos como mestizos. Los demás grupos minoritarios son: $2.34 \%$ blancos (4.5, incluyendo extranjeros que declaran ser blancos), $1.23 \%$ negro, $1.17 \%$ indígena, montubio $2.5 \%$ (el resto no describe autodefinición). El 3\% de los examinados representa algún tipo de discapacidad, el 14\% interrumpió en algún momento sus estudios y el $10 \%$ se cambió de universidad al menos una vez en algún momento de su trayectoria.

En cuanto a la forma de financiamiento de la colegiatura, el 68\% tuvo apoyo de sus padres, el $4 \%$ usó créditos educativos, $7 \%$ beca y el $12 \%$ autofinanció los estudios. En lo que respecta a la manutención durante el tiempo de estudios, el $70 \%$ tuvo apoyo de sus padres, $12 \%$ recursos propios, $10 \%$ de otros miembros del hogar. El 23\% trabajó en algún momento de su carrera. El promedio de miembros del hogar es de 5.13 personas, con 1.73 con una actividad económica remunerada. Esta descripción entra en el orden de los hogares de clase media ecuatoriana. En cuanto al índice de necesidades básicas en el hogar, el promedio es de 5.19/6. En cambio, en lo que respecta a bienes enseres baja a 2.58/5, pues debemos tener atención que en el orden de la pirámide de Maslow siguen los bienes básicos y luego los de seguridad.

Por último, la descripción de los jefes de hogar por escolaridad es la siguiente: El 47\% tiene educación superior completa y el 11\% alcanzó un posgrado, mientras el 12\% no terminó la universidad. Esto indica claramente que la mayoría de evaluados no son estudiantes universitarios de primera generación. La carrera de medicina se concentra en hogares de clase media con jefes de hogar educados (se debe considerar que la distribución de los ingresos en Ecuador y América Latina no es normal, por lo que la clase media no es mayoritaria) (ver anexo).

\section{Análisis de resultados}

De acuerdo con la literatura citada, en términos generales podemos hablar de una relación de causalidad o correlación positiva fuerte entre mejores condiciones socioeconómicas y éxito en los estudios, tomando en cuenta varios tipos de factores: quintiles de ingreso, grupos de migración, grupos étnicos menos favorecidos, ambiente familiar, trayectoria educativa del estudiante, educación familiar, etcétera.

Como se describió en la metodología, los modelos econométricos aplicados no arrojaron resultados significativos, por lo que hacemos una relación descriptiva entre las condiciones socioeconómicas, de acuerdo con el tipo de financiamiento de las universidades (particular, cofinanciada, pública).

En la Tabla 1 se puede observar que los promedios son bastante similares entre grupos de autodefinición étnica, con excepción del grupo montubio, que es muy inferior. Todos los grupos, con excepción del mestizo (ampliamente mayoritario) son inferiores a la media. Se hace una distinción en el grupo blanco total y de sólo ecuatorianos, porque buena parte de los examinados extranjeros se autodenominaron como blancos, y se quiso evidenciar las diferencias de este grupo con el de estudio (solo ecuatorianos).

$\mathrm{Si}$ se describe desde la perspectiva del tipo de financiamiento, los graduados de universidades públicas constituyen el $64.76 \%$ del total, $22.82 \%$ para cofinanciadas y $12.42 \%$ para privadas. La distribución de género por tipo de financiamiento es similar en todos los casos, por lo que, al parecer, no hay factores de género asociados al acceso a universidades por el tipo de financiamiento. 
Tabla 1. Promedios de calificación por autoidentificación étnica

\begin{tabular}{|c|c|c|c|c|c|c|}
\hline Promedio & Mestizos & Blancos total & $\begin{array}{c}\text { Blancos } \\
\text { universidades de } \\
\text { Ecuador }\end{array}$ & $\begin{array}{c}\text { Negros, } \\
\text { afroecuatorianos }\end{array}$ & Indigenas & Montubios \\
\hline 64.05 & 64.41 & 63.70 & 63.58 & 63.01 & 63.61 & 61.96 \\
\hline & $89.34 \%$ & $4.55 \%$ & $2.43 \%$ & $1.26 \%$ & $1.17 \%$ & $2.53 \%$ \\
\hline
\end{tabular}

Fuente: Encuesta de Evaluados EHEP 2017

Tabla 2. Distribución por tipo de financiamiento

\begin{tabular}{|l|c|c|c|}
\multicolumn{1}{|c}{ y género } \\
\hline & Estatal & Cofinanciada & Particular \\
\hline Hombres & 1231 & 430 & 230 \\
\hline Mujeres & 1836 & 651 & 358 \\
\hline Total & 3067 & 1081 & 588 \\
\hline Hombres & $40 \%$ & $40 \%$ & $39 \%$ \\
\hline Mujeres & $60 \%$ & $60 \%$ & $61 \%$ \\
\hline
\end{tabular}

Fuente: Encuesta de Evaluados EHEP 2017.

En la literatura sobre las condiciones socioeconómicas y el éxito educativo se suele resaltar cómo el ambiente familiar, la cultura de los padres y su trayectoria educativa inciden en el éxito o fracaso escolar (Coleman et al., 1966; Bourdieu, 1998; Deaton, 2015; Noguera, 2009). Las condiciones socioculturales mejoran o propician el éxito educativo. En el caso ecuatoriano, el nivel educativo de los padres funciona como un buen proxy para comprender que los estudiantes graduados de medicina pertenecen a familias de clase media que no son la primera generación universitaria. La sociología de Bourdieu (1998) suele resaltar cómo los valores simbólicos (capitales en términos del autor francés) que se precian más en un grupo son los que se suelen ser los que se busca reproducir y establecen trayectorias familiares. Valores culturales y simbólicos se "materializan" en su conversión en títulos universitarios; así, esta mayor valoración permite una continuidad que se traduce no sólo en el éxito educativo sino en las posibilidades de éxito profesional, medido en nuestro caso en la aprobación y calificaciones obtenidas en el examen de habilitación. Casi el 70\% de los jefes de hogar cuentan con algún tipo de estudios superiores y el 65\% de ellos completa. Un factor importante en los estudios de medicina de Ecuador es que hay una trayectoria intergeneracional en la decisión de estudiar medicina, los hijos suelen seguir los estudios de sus padres. 


\section{Gráfica 1. Nivel de escolaridad de los padres}

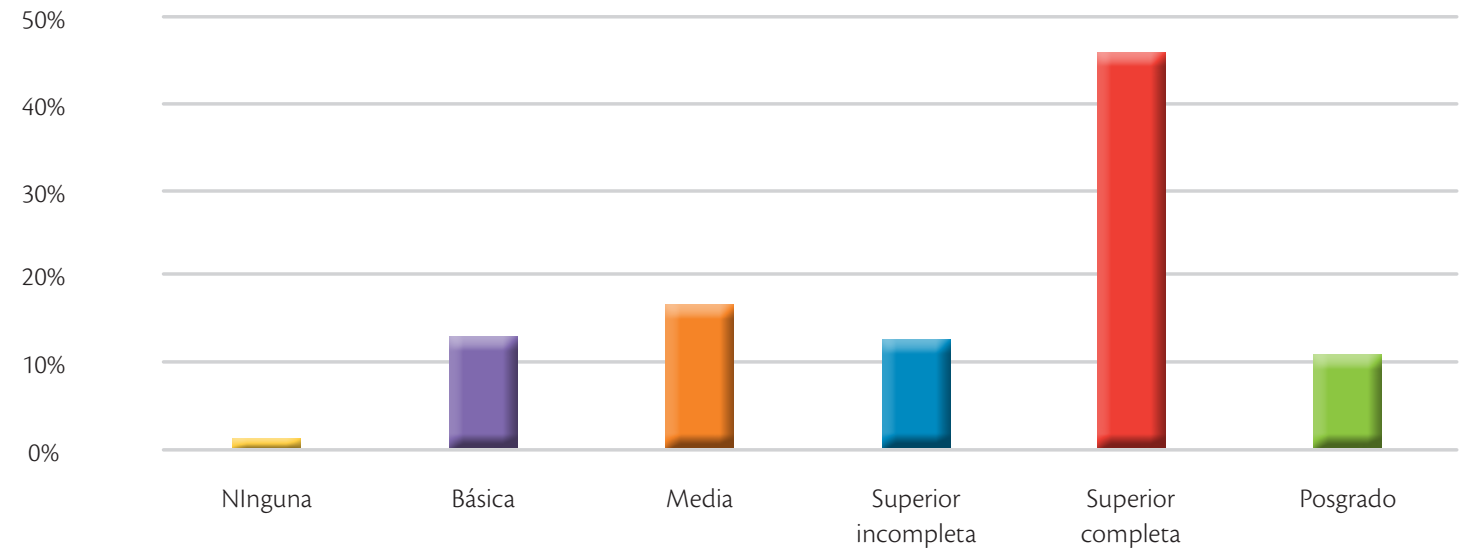

Fuente: Encuesta de Evaluados EHEP 2017.

Gráfica 2. Nivel de escolaridad de los jefes de hogar de acuerdo con el tipo de financiamiento de las universidades de los graduados de medicina

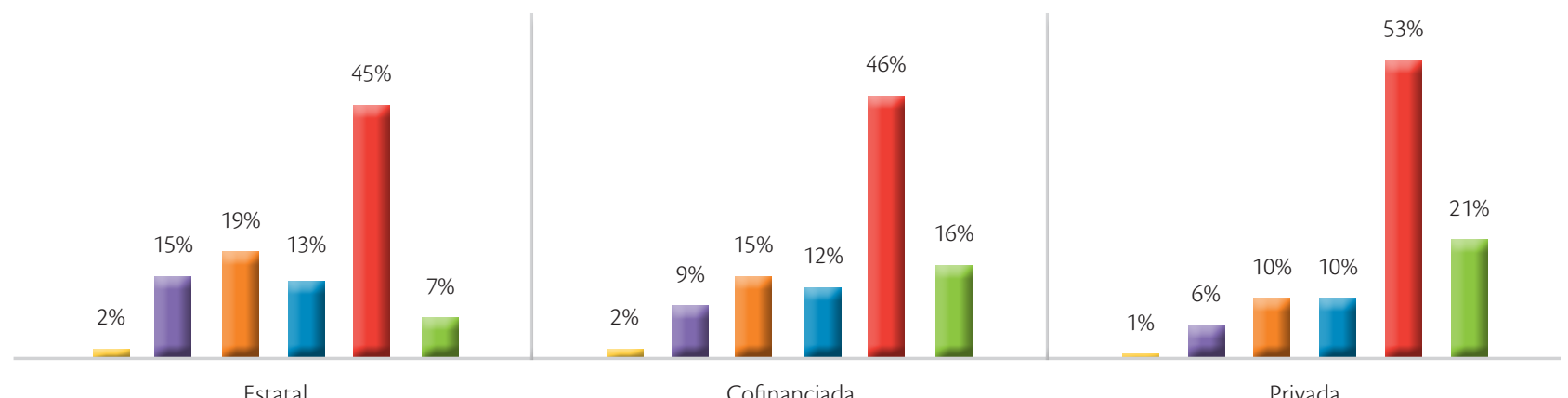

Si se observa desde la perspectiva del tipo de financiamiento de la universidad, se nota la misma tendencia general. Los graduados de medicina del Ecuador no son estudiantes universitarios de primera generación; pero sí se observan diferencias considerables de estudios superiores en las universidades privadas, en superior completa y acceso a posgrado.

Si el problema se analiza desde el tipo de financiamiento de las universidades, dividiéndolas en privadas, públicas y cofinanciadas, ya se marcan algunas diferencias. Los jefes de hogar con estudios de posgrado se concentran más en los estudiantes graduados de universidades privadas, al igual que quienes tienen estudios superiores completos. Jefes de hogar con algún tipo de educación superior en universidades públicas es del 65\%, en las cofinanciadas sube al $74 \%$, y en las privadas al $84 \%$. 
En lo que respecta a las necesidades básicas satisfechas, el índice va de 0 a 6 , como se explicó en la metodología. Se puede ver que entre las universidades cofinanciadas y privadas hay una gran paridad, y los porcentajes de acceso nulo o muy restringido (entre 0 y 2) a servicios básicos es de 0 o muy bajo. Evidentemente, el acceso universitario conlleva la resolución de problemas básicos de vivienda y servicios, incluso entre la población que se beneficia de la gratuidad de la educación. En este punto, se puede aclarar que en Ecuador hay una alta demanda para la carrera de medicina que suele ser muy costosa en universidades privadas y totalmente gratuita en las públicas. Por ejemplo, un estudiante de clase media alta podría apuntar a obtener un cupo de estudio en una universidad pública. Siguiendo la misma lógica de la literatura, si este estudiante proviene de colegios privados con mejor calidad de educación su éxito en el examen de ingreso es más probable, desplazando a una persona de clase baja. Probablemente, el mismo grupo social estudia medicina, priorizando hacerlo en una universidad pública y sólo en el caso de no obtenerlo ir a la universidad privada. Este tipo de explicación es plausible, pero parcial, porque sí hay diferencias entre privadas y cofinanciadas por un lado y públicas por otro. Mientras en el primer grupo de estudiantes, quienes alcanzan 5 o 6 en el índice son el $81 \%$ de los estudiantes, en las públicas llega al 76\%. Entonces, podría haber una combinación de ambos factores, por un lado, estudiantes con recursos podrían desplazar a otros debido al filtro de la prueba de ingreso en su acceso a las universidades públicas y, por otro, estudiantes de colegios privados podrían tener a universidades pagadas como su primera opción.

Esta diferencia más marcada ya se puede observar en cuanto al índice de acceso a bienes y enseres, que como se aclaró en la metodología, ya constituyen bienes más caros y sólo posibles una vez solucionados aquellos necesarios en el sentido de Maslow. Por ejemplo, entre quienes no tienen acceso a ningún tipo de bien descrito, hay un $14 \%$ en universidades públicas, frente al 6 y $4 \%$ en cofinanciadas y privadas. En cambio, en los índices 4 y 5 , hay $24 \%$ en las públicas, mientras en las cofinanciadas sube a $43 \%$ y en privadas a $52 \%$.

\section{Gráfica 3. Porcentaje del índice de necesidades básicas satisfechas distribuido por tipo de financiamiento de las universidades}

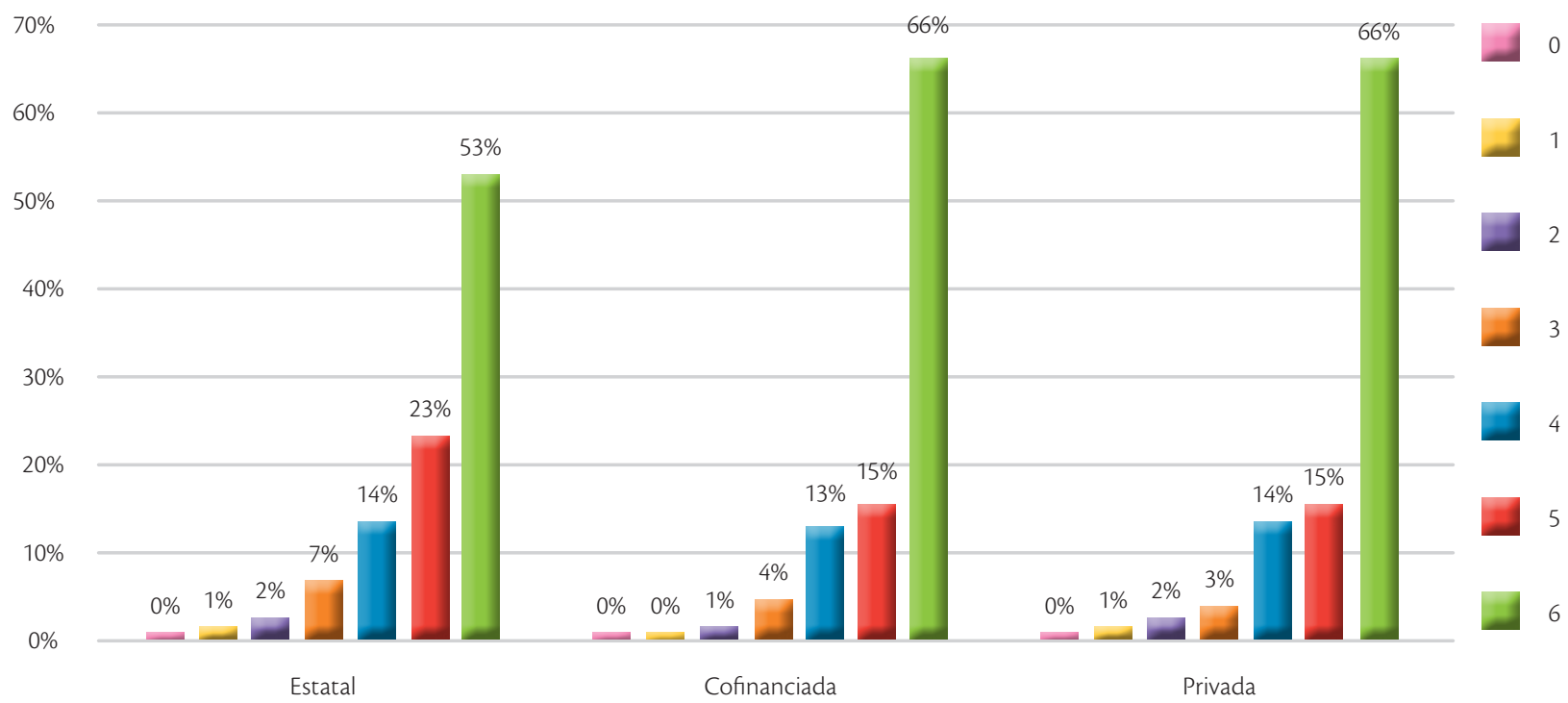




\section{Gráfico 4. Porcentaje del índice de acceso a bienes y enseres distribuidos por tipo de financiamiento de las universidades}

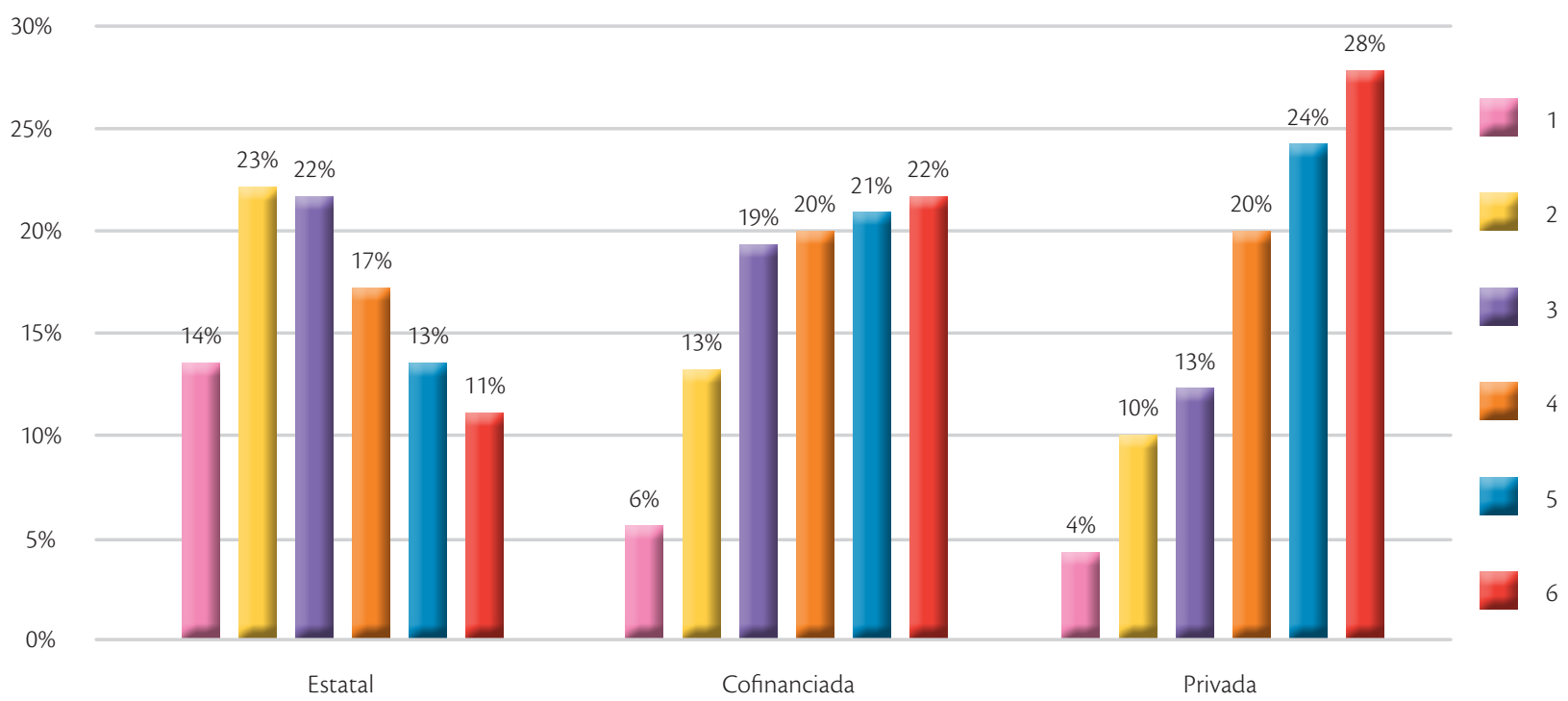

Fuente: Encuesta de Evaluados EHEP 2017.

Este tipo de bienes enseres no sólo favorece la propia educación, como en el caso de la conectividad o los ordenadores, también la movilidad, ocio y recreación, mejorando el nivel de vida y el propio acceso a fuentes diversas de conocimiento. El sistema de enseñanza en universidades privadas, conocido como Aprendizaje Basado en Problemas (ABP), demanda el acceso a fuentes de información calificadas para que los estudiantes puedan construir los casos clínicos con evidencia de diversas fuentes.

La prueba de diferencia de medias arroja datos interesantes sobre la hipótesis que maneja la literatura.
Desde la perspectiva de los promedios de los estudiantes de las universidades públicas con respecto a las privadas y cofinanciadas, existen diferencias significativas en los promedios, pero si se compara entre universidades privadas y cofinanciadas, esta diferencia estadística desaparece, lo que confirma una relación entre las condiciones socioeconómicas y mayores probabilidades de éxito educativo y también profesional medido en los exámenes de habilitación. Siguiendo el índice de acceso a bienes enseres, sí hay diferencias claras entre los graduados de universidades públicas y de universidades pagadas. 
Tabla 3. Diferencia de medias entre promedios de universidades por tipo de financiamiento

\begin{tabular}{|c|c|c|c|c|c|c|}
\hline & Observaciones & Media & Error estándar & $\begin{array}{l}\text { Desviación } \\
\text { estándar }\end{array}$ & $95 \%$ conf & Intervalo \\
\hline Pública & 3062 & 65.51765 & 0.173544 & 9.603140 & 62.1774 & 65.858 \\
\hline Cofinanciada & 1082 & 68.88632 & 0.313505 & 10.31230 & 68.2712 & 69.505 \\
\hline Combinados & 4144 & 66.39720 & 0.153839 & 9.90320 & 66.0956 & 66.699 \\
\hline Diferencia & & -3.368686 & 0.358334 & & -4.07148 & -2.6658 \\
\hline Ho dif $=0$ & & & & & & $t=-9.4010$ \\
\hline Ha dif $<0$ & & & Ha med $!=0$ & & & Ha med $>0$ \\
\hline \multirow[t]{2}{*}{$\mathrm{T}<\mathrm{t}=0.000$} & & & 0.0000 & & & $\mathrm{~T}>\mathrm{t}=1.000$ \\
\hline & Observaciones & Media & Error estándar & $\begin{array}{l}\text { Desviación } \\
\text { estándar }\end{array}$ & $95 \%$ conf & Intervalo \\
\hline Pública & 3062 & 65.51764 & 0.173545 & 9.60314 & 65.1774 & 65.858 \\
\hline Privada & 581 & 69.58348 & 0.391975 & 9.44815 & 68.8136 & 70.353 \\
\hline Combinados & 3643 & 66.16607 & 0.160583 & 9.69234 & 65.8512 & 66.481 \\
\hline Diferencia & & -4.06584 & 0.428675 & & -4.90727 & -3.2244 \\
\hline Ho dif $=0$ & & & & & & $t=-9.4847$ \\
\hline Ha dif $<0$ & & & Ha med $!=0$ & & & Ha med $>0$ \\
\hline \multirow[t]{2}{*}{$\mathrm{T}<\mathrm{t}=0.0000$} & & & 0.0000 & & & $\mathrm{~T}>\mathrm{t}=1.000$ \\
\hline & Observaciones & Media & Error estándar & $\begin{array}{l}\text { Desviación } \\
\text { estándar }\end{array}$ & $95 \%$ conf & Intervalo \\
\hline Privada & 581 & 69.58348 & 0.391975 & 9.44815 & 68.8136 & 70.3534 \\
\hline Cofinanciada & & 68.88632 & 0.313505 & 10.31230 & 68.2718 & 69.5015 \\
\hline Combinados & 1038 & 69.12989 & 0.245748 & 10.02160 & 68.6479 & 69.6119 \\
\hline Diferencia & & 0.697155 & 0.501927 & & -0.28753 & 1.68185 \\
\hline Ho dif $=0$ & & & & & & $t=1.3890$ \\
\hline Ha dif $<0$ & & & Ha med $!=0$ & & & Ha med $>0$ \\
\hline $\mathrm{T}<\mathrm{t}=0.9175$ & & & 0.1651 & & & $T>t=0.0825$ \\
\hline
\end{tabular}

Fuente: Encuesta de Evaluados EHEP 2017. 


\section{Conclusiones}

Con base en la literatura que vincula factores socioeconómicos, culturales y de nivel educativo, este estudio intentó establecer una relación entre el éxito de los graduados de medicina en el examen de habilitación para el ejercicio profesional. La propia aplicación al examen constituye ya un punto culminante de estudios previo al ejercicio profesional, en el que se han salvado ya obstáculos y filtros, relacionados a las condiciones socioeconómicas, culturales y motivacionales.

Sobre los hallazgos, se puede observar que el índice de acceso a servicios y bienes básicos muestra menos diferencias que el de bienes y enseres del hogar. El índice de acceso a bienes enseres es mucho más favorable entre universidades privadas y cofinanciadas respecto a las públicas. De la misma manera, haciendo un análisis de diferencia de medias se puede ver que los promedios obtenidos en universidades públicas tienen diferencias estadísticas significativas hacia abajo que las que muestran las universidades privadas y cofinanciadas. En este sentido, se pueden deducir varias cosas: si el acceso a servicios básicos no difiere entre los grupos de estudiantes de acuerdo con el tipo de financiamiento de la universidad, se sigue que para poder graduarse en una universidad en una carrera larga como medicina, se debe haber solventado al menos lo básico; hay un mínimo requerido para el primer éxito educativo. Sin embargo, si se mide en cuanto a las posibilidades de éxito profesional de acuerdo con la nota obtenida en el EHEP, se necesita algo más y los grupos se van separando poco a poco, de acuerdo con las ventajas por el acceso a bienes enseres. Esto implica que los círculos virtuosos se reproducen no sólo académica sino también profesionalmente. Para llegar a ser estudiante universitario y graduarse no se puede ser pobre, y mientras menos pobre, más probabilidades de éxito profesional.

En cuanto a la autoidentificación étnica, no hay variaciones significativas, además si se toma en cuenta que la gran mayoría se ubica como mestizo. El único grupo que se separa de la media son aquellos autoidentificados como montubios. En lo que respecta al apoyo familiar económico y a la tradición familiar de estudios, se evidencia que la carrera de medicina es mayoritariamente sustentada por los padres o el núcleo familiar (sobre todo, tomando en cuenta que se trata de una carrera muy larga) y que la mayoría de los graduados de medicina no son profesionales universitarios de primera generación. Si se analiza ya a los grupos de estudiantes por tipo de financiamiento sí se evidencia que la mayoría de jefes de hogar con algún tipo de estudios universitarios, graduados o con posgrados, se encuentran entre los examinados de universidades privadas o cofinanciadas. El entorno familiar es muy importante no sólo en términos de sostén económico, sino también en el valor simbólico otorgado a obtener una carrera universitaria. En cuanto al intento del acercamiento al problema con un modelo econométrico binario para aprobados y no aprobados, surgen varias interrogantes acerca de la consistencia del propio examen y el tipo de aptitudes que mide. Una opción importante a considerar es que el examen mide mejor las aptitudes relacionadas con un tipo de estudios que se desarrolla en universidades privadas y cofinanciadas, llamado Aprendizaje Basado en Problemas (ABP), sólo posibles con grupos de pocos alumnos, imposible en universidades públicas. Otra cuestión esencial es observar cómo se estructura la matrícula en el país, para la cual ya hay filtros de acceso por exámenes. Como aseveran Ponce y Carrasco (2016), en Ecuador los filtros de los exámenes de ingreso a las universidades pueden jugar en contra del propio acceso y de la gratuidad. Dado que se trabaja con personas ya graduadas - múltiples filtros - podría haber menos incidencia de las variables socioeconómicas para hacer un modelo de regresión.

En general, se ratifica la idea de las ventajas de los factores socioeconómicos, culturales, trayectoria educativa de los padres, y forma de estudios en universidades privadas y cofinanciadas para el éxito en el EHEP, lo que puede determinar mayores posibilidades de éxito profesional. 


\section{Referencias}

Becker, G. (1994), Human capital: a theoretical and empirical analysis with special reference to education, Chicago, University Chicago Press.

Blanden, J. y P. Gregg (2004), "Family income and educational attainment: a review of approaches and evidence for Britain", Oxford Review of Economic Policy, vol. 20, núm. 2, pp. 245-263, DOI: https://doi. org/10.1093/oxrep/grh014.

Brint, S. y Ch. Clotfelter (2016), "U.S. higher education effectiveness", Journal of the Social Sciences, vol. 2, núm. 1, pp. 2-37.

Bourdieu, P. (1998), La distinción. Criterios y bases sociales del gusto, Madrid, Taurus.

Cadil, Jan, L. Petkovová y D. Blatná (2014), "Human capital, economic structure and growth", Procedia Economics and Finance, núm. 12, pp. 85-92, DOI: doi. org/10.1016/S2212-5671(14)00323-2.

Carrillo Álvarez, E., M. Civís Zaragoza, T. Blanch, E. Longás Mayayo y J. Riera Romaní (2018), "Condicionantes del éxito y fracaso escolar en contextos de bajo nivel socioeconómico", REXE: Revista de estudios y experiencias en educación, vol. 2, núm. 1, pp. 75-94.

Chiroleu, A. (2009), "La inclusión en la educación superior como política pública: tres experiencias en América Latina”, Revista Iberoamericana de Educación, núm. 48, pp. 1-15.

Clark, M.A., M. Brooks, S. Lee, L. Pasquarella Daley, Y. Crawford y S. Maxis (2006), "Factors influencing the educational success of minority pre-service educators", Journal of College Student Retention: Research, Theory \& Practice, vol. 8, núm. 1, DOI: doi.org/10.2190/9HJ4LXCU-B40X-B35M.

Coleman, J. S. (1968), "Social capital in the creation of human capital", The American fournal of Sociology, núm. 94, pp. S95-S120.

Coleman, J., E. Q. Campbell, C.J. Hobson, J. Mc Partland, A. M. Mood, F. D. Weinfeld y R. York (1966), Equality of educational opportunity, Washington, Office of Education.

Cordero Ferrara, J. M., F. Pedraja Chaparro, R.
Simancas Rodríguez (2015), "Factores del éxito escolar en condiciones socioeconómicas desfavorables", Revista de Educación, núm. 370, pp. 172-198, DOI: 10.4438/1988-592X-RE-2015-370-302.

Crisp, G., Taggart, A., Nora, A. (2005). "Undergraduate Latina/o Students: A Systematic Review of Research Identifying Factors Contributing to Academic Success Outcomes", Review of Educational Research, vol. 85, núm. 2, pp. 249-274, DOI: https://doi. org/10.3102/0034654314551064.

Deaton, A. (2015), El gran escape: salud, riqueza y los orígenes de la desigualdad, México, FCE.

Enríquez, O. y B. Mena (2005), "Habilitación profesional. Condiciones para el aseguramiento de la calidad de la educación médica y condiciones para la confianza recíproca. Experiencia y visión de ASOFAMECH", Revista Médica de Chile, núm. 133, pp. 483-494.

Escobar-Jiménez, C. y A. Delgado (2019), "Calidad y cualidad en la educación superior: una discusión teórica en el sistema de educación superior ecuatoriano", REDU. Revista de Docencia Universitaria, vol. 17, núm. 2, pp. 159175, DOI: https://doi.org/10.4995/redu.2019.11740.

García de Fanelli, A. y C. Jacinto (2010), "Equidad y educación superior en América Latina: el papel de las carreras terciarias y universitarias", Revista Iberoamericana de Educación Superior, vol. 1, núm. 1, pp. 58-75.

Harris, D. N. (2007), "High-flying schools, student disadvantage and the logic of NCLB", American fournal of Education, vol. 113, núm. 3, pp. 367-394.

Harris III, J., M. Harris Pyles, D. G. Carter (1976), "The culturally different: an analysis of factors influencing educational success", The Clearing House: A Journal of Educational Strategies, Issues and Ideas, vol. 50, núm. 1, pp. 39-43, DOI: 10.1080/00098655.1976.9956901.

Lorenzo Moledo, M. M., M. Santos Rego y A. Godás Otero (2012), “Inmigración y educación. ¿influye el nivel educativo de los padres en el rendimiento académico de los hijos?", Teoría de la Educación: Revista Interuniversitaria, vol. 24, núm. 2. 
Noguera, P. (2003), "The trouble with black boys: the role and influence of environmental and cultural factors on the academic performance of African American males", Urban Education, vol. 25, núm. 4, pp. 431-459, DOI: doi.org/10.1177/0042085903038004005.

Noguera, P. (2009), The trouble with black boys:... And other reflections on race, equity, and the future of public education, New York, John Wiley \& Sons.

O’Neill, L. D., B. Wallstedt, B. Eika y J. Hartvigsen (2011), "Factors associated with dropout in medical education: a literature review", Medical Education, núm. 45, pp. 440454, DOI: doi:10.1111/j.1365-2923.2010.03898.x.

Pàmies Rovira, J. (2011), "Éxito académico, inmigración y ciudadania. Condiciones y posibilidades entre jóvenes de origen marroquí en Cataluña", en F. J. García Castaño y N. Kressova (coords.), Actas del I Congreso Internacional sobre Migraciones en Andalucia, Granada, Instituto de Migraciones, pp. 443-448.

Ponce, J. y F. Carrasco (2016), "Acceso y equidad a la educación superior y posgrado en el Ecuador, un enfoque descriptivo", Mundos plurales, Revista Latinoamericana de Políticas y Acción Pública, vol. 3, núm. 2, pp. 9-22.

Rama, C. (2009), "La tendencia a la masificación de la cobertura de la educación superior en América Latina", Revista Iberoamericana de Educación Superior, vol. 50, pp. 173-195.

Ruano, A. L., A. Vizuete, J. Moreno y W. Quishpe
(2018), "Habilitación profesional: caso Ecuador", Revista Ecuatoriana de Medicina Eugenio Espejo, vol. 7, núm. 9, DOI: https://doi.org/10.23936/ree.v7i9.16.

Scott, J., K. Taylor y R. Palmer (2013), "The race against time: preparing black students for the changing landscape of higher education", The Fournal of Negro Education, vol. 82, núm. 3, pp. 288-299.

Subhiyah, R. (2018), "Standards of an examination (cut points), construction of scenarios, measurement of impacts in the decision making process", Revista Ecuatoriana de Medicina Eugenio Espejo, vol. 7, núm. 9, DOI: https://doi.org/10.23936/ree.v7i9.16.

Sverdlik, I., P. Ferrara y A. Jaimovich (2005), Desigualdad e inclusión en la educación superior. Un estudio comparado en cinco países de América Latina, Buenos Aires, OPED.

Victora, C., B. Lessa Horta, C. Loret de Mola, L. Quevedo, R. Tavares Pinheiro, D. Gigante, H. Goncalves, F. Barros (2015), "Association between breastfeeding and intelligence, educational attainment, and income at 30 years of age: a prospective birth cohort study from Brazil", The Lancet Global Health, vol. 3, núm. 4, pp. 199-205, DOI: https://doi.org/10.1016/ S2214-109X(15)70002-1.

Yang, Y. y J. E. Gustafsson (2004), "Measuring socioeconomic status at individual and collective levels", Educational Research and Evaluation, vol. 10, núm. 3, pp. 259-288. 
Anexo 1

\begin{tabular}{|c|c|c|c|c|}
\hline Variable & Media & $\begin{array}{l}\text { Desviación } \\
\text { estándard }\end{array}$ & Min & $\operatorname{Max}$ \\
\hline \multicolumn{5}{|l|}{ Variables dependientes } \\
\hline Calificación & 66.45 & 6.54 & 32 & 87 \\
\hline Resultado (aprobado) & 0.746 & 0.44 & 0 & 1 \\
\hline \multicolumn{5}{|c|}{ Características de los estudiantes } \\
\hline Género (femenino) & 0.61 & 0.49 & 0 & 1 \\
\hline Año de nacimiento & 1988 & 3.77 & 1957 & 1994 \\
\hline \multicolumn{5}{|l|}{ Etnia: } \\
\hline Mestizo & 0.89 & 0.23 & 0 & 1 \\
\hline Blanco & 0.05 & 0.14 & 0 & 1 \\
\hline Negro & 0.01 & 0.17 & 0 & 1 \\
\hline Indígena & 0.01 & 0.12 & 0 & 1 \\
\hline Discapacidad & 0.03 & 0.2 & 0 & 1 \\
\hline Cambio de universidad & 0.10 & 0.27 & 0 & 1 \\
\hline Interrupción estudios & 0.14 & 0.36 & 0 & 1 \\
\hline \multicolumn{5}{|l|}{ Financiamiento escolaridad: } \\
\hline Propio & 0.12 & 0.48 & 0 & 1 \\
\hline Padre o tutores & 0.68 & 0.49 & 0 & 1 \\
\hline Pareja & 0.02 & 0.1 & 0 & 1 \\
\hline Hermanos & 0.05 & 0.17 & 0 & 1 \\
\hline Otros miembros del hogar & 0.02 & 0.15 & 0 & 1 \\
\hline Beca & 0.07 & 0.18 & 0 & 1 \\
\hline Crédito & 0.04 & 0.13 & 0 & 1 \\
\hline \multicolumn{5}{|l|}{ Financiamiento manutención: } \\
\hline Propio & 0.12 & 0.32 & 0 & 1 \\
\hline Padre o tutores & 0.70 & 0.29 & 0 & 1 \\
\hline Pareja & 0.03 & 0.18 & 0 & 1 \\
\hline Hermanos & 0.05 & 0.18 & 0 & 1 \\
\hline Otros miembros del hogar & 0.02 & 0.12 & 0 & 1 \\
\hline
\end{tabular}




\begin{tabular}{|c|c|c|c|c|}
\hline Otros familiares & 0.04 & 0.18 & 0 & 1 \\
\hline Beca & 0.02 & 0.1 & 0 & 1 \\
\hline Crédito & 0.02 & 0.13 & 0 & 1 \\
\hline Trabajo en el transcurso de la carrera & 0.23 & 0.42 & 0 & 1 \\
\hline \multicolumn{5}{|l|}{ Características del hogar } \\
\hline Tamaño hogar & 5.13 & 1.72 & 1 & 20 \\
\hline Miembros hogar con actividad económica & 1.74 & 0.87 & 0 & 10 \\
\hline \multicolumn{5}{|l|}{ Enseres del hogar: } \\
\hline Lavadora & 0.61 & 0.5 & 0 & 1 \\
\hline Automóvil & 0.45 & 0.49 & 0 & 1 \\
\hline TV pantalla plana & 0.37 & 0.44 & 0 & 1 \\
\hline Computadora de escritorio & 0.69 & 0.47 & 0 & 1 \\
\hline Smartphone & 0.46 & 0.46 & 0 & 1 \\
\hline Conexión a internet & 0.59 & 0.5 & 0 & 1 \\
\hline \multicolumn{5}{|c|}{ Características de las necesidades básicas del hogar: } \\
\hline Paredes adobe/tapia & 0.02 & 0.13 & 0 & 1 \\
\hline Paredes asbesto/cemento & 0.11 & 0.32 & 0 & 1 \\
\hline Paredes caña revestida/carrizo & 0 & 0.04 & 0 & 1 \\
\hline Paredes caña no revestida & 0 & 0.07 & 0 & 1 \\
\hline Paredes hormigón/bloque/ladrillo & 0.83 & 0.37 & 0 & 1 \\
\hline Paredes madera & 0.02 & 0.15 & 0 & 1 \\
\hline Piso cemento & 0.3 & 0.46 & 0 & 1 \\
\hline Piso cerámica & 0.56 & 0.5 & 0 & 1 \\
\hline Piso marmol & 0.03 & 0.18 & 0 & 1 \\
\hline Piso tabla & 0.09 & 0.29 & 0 & 1 \\
\hline Piso tierra & 0.01 & 0.12 & 0 & 1 \\
\hline Excusado conectado a alcantarillado & 0.88 & 0.35 & 0 & 1 \\
\hline Agua potable red pública por tubería & 0.93 & 0.27 & 0 & 1 \\
\hline
\end{tabular}




\begin{tabular}{|c|c|c|c|c|}
\hline Electricidad & 0.96 & 0.18 & 0 & 1 \\
\hline Îndice necesidades básicas cubiertas & 5.19 & 1.74 & 0 & 5 \\
\hline Îndice enseres del hogar & 2.58 & 1.9 & 0 & 6 \\
\hline \multicolumn{5}{|l|}{ Características del jefe de hogar } \\
\hline \multicolumn{5}{|l|}{ Escolaridad jefe de hogar: } \\
\hline Sin escolaridad & 0.01 & 0.14 & 0 & 1 \\
\hline Educación básica & 0.12 & 0.35 & 0 & 1 \\
\hline Educación secundaria & 0.187 & 0.38 & 0 & 1 \\
\hline Superior completa & 0.47 & 0.29 & 0 & 1 \\
\hline Superio incompleta & 0.12 & 0.21 & 0 & 1 \\
\hline Postgrado & 0.11 & 0.29 & 0 & 1 \\
\hline
\end{tabular}

\section{Cómo citar este artículo:}

Escobar-Jiménez, Christian y Sergio Torres-Rentería (2021), "Éxito educativo y condiciones socioeconómicas: los exámenes de habilitación para ejercer la medicina en Ecuador", Revista Iberoamericana de Educación Superior (RIES), vol. XII, núm. 35, pp. 132-149, DOI: https://doi.org/10.22201/iisue.20072872e.2021.35.1086 [Consulta: fecha de última consulta]. 